\title{
ANÁLISE COMPARATIVA DOS GUIAS ALIMENTARES PARA A POPULAÇÃO BRASILEIRA E ARGENTINA
}

\section{Maria Devany Pereira}

Graduação em Nutrição pela Universidade Federal do Piauí, UFPI, Brasil.

\section{Alana Paulina de Moura Sousa}

Graduação em Nutrição pela Universidade Federal do Piauí, UFPI, Brasil.

\section{Janekeyla Gomes de Sousa}

Nutricionista no Instituto Federal de Educação, Ciência e Tecnologia do Rio Grande do Norte, IFRN, Brasil.

\section{Patricia Maria Beatriz Perricone}

Universidade Nacional da Catamarca, UNCA, Argentina.

\section{Cecília Maria Resende G. de Carvalho}

Doutorado em Alimentos e Nutrição pela Universidade Estadual de Campinas, UNICAMP, Brasil.

\section{Marize Melo dos Santos}

Docente na Universidade Federal do Piauí, UFPI, Brasil. Doutorado em Nutrição pela Universidade Federal de Pernambuco, UFPE, Brasil.
RESUMO: Vários países já desenvolveram seus guias com objetivos semelhantes, porém, abrangendo suas particularidades. Objetivou-se analisar o Guia Alimentar para a População Brasileira e o Guia Alimentar para a População Argentina, identificando possíveis semelhanças e diferenças entre estes, relacionando-os quanto à estrutura eà promoção de práticas alimentares saudáveis. Trata-se do estudo descritivo que utilizou o Guia Alimentar Brasileiro e Argentino, com levantamento bibliográfico e análise documental quanto às principais diferenças e semelhanças estruturais e de desenvolvimento. Ao comparar os guias alimentares, observou-se maior conteúdo técnico no guia argentino. Enquanto o brasileiro apresenta as informações de forma mais resumida e dinâmica. Ambos chamam a atenção para a importância da promoção de práticas alimentares saudáveis em todas as fases da vida. A melhoria da qualidade e o aprimoramento dos guias alimentares são fundamentais para a qualidade de vida da população. Logo, maiores estudos que abordem o entendimento e o ponto de vista da população sobre o guia alimentar fazem-se necessários.

PALAVRAS-CHAVE: Guias alimentares; Promoção da saúde; Brasil; Argentina; Saúde pública.

\section{COMPARATIVE ANALYSIS OF DIET GUIDELINES FOR BRAZILIANS AND ARGENTINES}

\begin{abstract}
Several countries have prepared guidelines with similar aims, albeit featuring their peculiarities. Current study analyzes the Food Guidelines for Brazilians and Argentines, identifying possible similarities and differences and relating them to structures and promotion of health food practices. Current descriptive study uses the Brazilian and Argentine Food Guide, bibliographic survey and documental analysis with regard to the main differences and similarities, structures and their development. A comparison of the food guides reveals high technical contents in the Argentine book, whilst the Brazilian guide provides dynamic and short information. The two guides focus on the promotion of healthy food practices in all life stages. Quality improvement in food guides is basic for population's life quality. Further studies on the understanding and point of view of the population on food guides are required.
\end{abstract}

KEY WORDS: Food Guides; Health Promotion; Brazil; Argentina; Public Health. 


\section{INTRODUÇÃO}

Guias alimentares são instrumentos de promoção à saúde, os quais variam de acordo com o país, possuindo formatos, números, porções e grupos alimentares diferentes, porém todos têm o mesmo objetivo, transformar o conhecimento científico de nutrição em conceitos básicos para que grande parcela da população seja orientada quanto à forma de se alimentar adequadamente ${ }^{1}$.

A World Health Organization - WHO lançou a Estratégia Global (EG) sobre dieta, atividade física e saúde, em 2004, apoiada também pelo Ministério da Saúde (MS) do Brasil, que em 2008 lançou o Guia Alimentar para a População Brasileira (GAPB), como ferramenta da Política Nacional para Alimentação e Nutrição (PNAN) ${ }^{2,3}$.

A segunda edição do GAPB foi publicada em 2014 pelo MS, visto o cenário atual do Brasil, sendo importante instrumento de apoio às ações de educação alimentar e nutricional. Dentre as principais recomendações estão: priorizar o consumo de alimentos frescos (in natura ou minimamente processados) e preparações culinárias em detrimento ao consumo de alimentos ultraprocessados ${ }^{4}$.

O Guia Alimentar para a População Argentina (GAPA) é uma ferramenta que combina conhecimentos científicos avançados com estratégias educativas, que contribui para gerar conhecimento e compreensão do comportamento nutricional mais equilibrado e saudável pela população de usuários diretos e indiretos. O principal objetivo é promover um estilo de vida mais saudável, a fim de prevenir problemas de saúde relacionados à dieta, a partir de uma abordagem baseada em alimentos. Para a elaboração das diretrizes, foi contemplada a situação nutricional-epidemiológica de todo o país com forte predominância de doenças crônicas não transmissíveis 5 .

Uma alimentação saudável está associada à redução da obesidade e dos riscos provocados em todas as fases da vida. Dessa forma, melhorar a qualidade da dieta desde a adolescência nos permite construir melhorias no futuro da população $0^{6}$.

O projeto "Estudo de práticas de vida saudável com estudantes de ensino fundamental e médio na perspectiva da promoção da soberania e segurança alimentar e nutricional", parte da Rede SSAN (Soberania e Segurança Alimentar e Nutricional), faz conexão entre a Universidade Federal do Piauí - UFPI e a Universidad Nacional de Catamarca - UNCA, cuja perspectiva é desenvolver ações e diagnóstico de estilo de vida saudável com estudantes de ensino fundamental na perspectiva da promoção da soberania e segurança alimentar e nutricional.

À luz deste contexto, o presente artigo tem como objetivo analisar o GAPB e GAPA, identificando possíveis semelhanças e diferenças entre estes, relacionando-os quanto à estrutura e à promoção de práticas alimentares saudáveis.

\section{METODOLOGIA}

Trata-se de um estudo descritivo de base primária. Foram analisados o GAPB (2014) e o GAPA (2016), visto a parceria Rede SSAN entre as Universidades do Piauí Brasil e da Catamarca - Argentina, na realização de um projeto de pesquisa intitulado "Estudo de práticas de vida saudável com estudantes de ensino fundamental e médio na perspectiva da promoção da soberania e segurança alimentar e nutricional".

Para organização dos dados foi realizado levantamento bibliográfico a fim de identificar publicações para embasar a discussão deste artigo, comparando os guias alimentares destes países. A revisão da literatura e análise do conteúdo dos guias ocorreu no período entre novembro de 2018 e fevereiro de 2019.

Para elaboração da análise documental dos guias utilizou-se a abordagem semelhante a Andrade e Docca ${ }^{7}$, resultando na construção das seguintes categorias: principais diferenças estruturais e desenvolvimento, pontos positivos, negativos e abordagem da promoção de práticas alimentares saudáveis.

\section{RESULTADOS}

\section{GUIA ALIMENTAR PARA POPULAÇÃO BRASILEIRA}

O GAPB, lançado em 2014, passou por um processo de consulta pública, permitindo a construção de uma versão de acordo com as mudanças no perfil de saúde e estilo de vida da população brasileira. O GAPB 
está estruturado em preâmbulo e introdução, com cinco capítulos (princípios; a escolha dos alimentos; dos alimentos à refeição; o ato de comer e a comensalidade; a compreensão e a superação de obstáculos) seguido dos "Dez passos para uma alimentação adequada e saudável", uma seção intitulada "Para saber mais" e "Anexos" explicando o processo de elaboração.

$\mathrm{O}$ preâmbulo apresenta de forma sucinta $\mathrm{o}$ porquê e a importância do guia, que é um marco na estratégia de implementação da diretriz de promoção da alimentação adequada e saudável que integra a PNAN, apoiando e incentivando práticas alimentares saudáveis individual e coletivamente. Na introdução contém resumido o que será encontrado no GAPB, relatando brevemente os capítulos que se seguem, com o objetivo de ser divulgado para todos os brasileiros e utilizado em todo e qualquer espaço onde ocorram atividades de promoção da saúde.

O primeiro capítulo apresenta os cinco princípios que orientaram a elaboração do GAPB; primeiramente mostra a importância da combinação de nutrientes, alimentos, preparações culinárias e as dimensões culturais e sociais das práticas alimentares, assim como as orientações alimentares devem levar em consideração as mudanças do perfil de alimentação e hábitos de vida dos brasileiros. A alimentação adequada e saudável deriva de sistema alimentar socialmente e ambientalmente sustentável, sendo desenvolvido a partir de diferentes saberes e contribuindo para a ampliação da autonomia nas escolhas alimentares.

De acordo com o GAPB, as orientações e as recomendações foram desenvolvidas utilizando os conhecimentos produzidos por estudos experimentais, clínicos, populacionais e antropológicos, bem como foi levado em consideração os conhecimentos implícitos na formação dos padrões tradicionais de alimentação.

No segundo capítulo são descritas as escolhas dos alimentos, trazendo quatro categorias definidas de acordo com o tipo de processamento empregado na sua produção. O tipo de processamento influencia no teor de nutrientes, no gosto dos alimentos, além de influir na escolha dos demais alimentos que serão consumidos, nas circunstâncias em que serão consumidos e na quantidade. Cada categoria de processamento vem ilustrada com os principais alimentos do grupo, citando exemplos e "o que são".

O primeiro grupo, o qual se orienta ser a base da alimentação, são os in natura ou minimamente processados, trata-se de alimentos que não sofram ou tenha um mínimo de alteração após ser obtido da natureza, tendem a se deteriorar muito rapidamente em decorrência do mínimo processamento. Óleos, gorduras, sal e açúcar constituem o segundo grupo, devendo ser utilizados com moderação em preparações culinárias, pelo seu alto teor de nutrientes que quando consumidos em excesso aumenta o risco de doenças crônicas.

Seguindo, têm-se os alimentos processados que devem ser consumidos limitadamente, pela adição de sal, açúcar e conservantes, como conservas de legumes, compota de frutas, queijos e pães. E por fim, o grupo dos ultraprocessados que se recomenda evitar o consumo pelos vários processos para obter o produto final, afetando de modo desfavorável a cultura, a vida social e o meio ambiente da população. Ao final do capítulo tem-se a "Regra de ouro" que é para orientar a preferência sempre pelos alimentos in natura ou minimamente processados e preparações culinárias a alimentos ultraprocessados. A 'regra de ouro' resume as orientações passadas sobre os tipos de alimentos.

Oterceirocapítuloexplicadesdeasrecomendações dos alimentos à refeição, complementando o capítulo anterior, com fotos de preparações culinárias que buscam representar homens e mulheres a partir de dez anos de idade, desde o café da manhã, almoço, jantar e pequenas refeições (lanches), levando em consideração a alimentação das diferentes regiões do país, meio urbano e rural e diferentes rendas. Têm-se também exemplos dos principais grupos que constituem a alimentação da maioria dos brasileiros (feijões, cereais, raízes, tubérculos, legumes e verduras, frutas, castanhas, leite, carnes e ovos, água), mostrando como escolher, conservar e manipular estes alimentos.

O próximo e quarto capítulo expõe o ato de comer e a comensalidade, que apresenta três orientações básicas: comer com regularidade e com atenção; comer em ambientes apropriados; e comer em companhia. O capítulo ilustra diferentes famílias, realizando suas refeições juntas de forma agradável, demonstrando a 
importância e todo o contexto que abrange o ato de comer e influência direta na saúde e bem-estar das pessoas.

A compreensão e a superação de obstáculos apresentado no capítulo 5 , com pontos que influenciam e estão ligados diretamente à atitude alimentar das pessoas, tópicos como informações não confiáveis, problemas relacionados à oferta de alimentos, o custo, a habilidade culinária, a falta de tempo das pessoas e a publicidade em particular para crianças e adolescentes.

O GAPB apresenta, ainda, um tópico com "10 passos para uma alimentação adequada e saudável”, resumindo as orientações neste presente, como escolhas alimentares saudáveis em ambientes apropriados e com companhia agradável, devendo ter atenção com os locais de compra dos alimentos e propagandas comerciais. Os últimos tópicos possuem sugestões de leituras adicionais que aprofundam os temas abordados e discutidos em cada um dos capítulos e anexo mostra como foi construído este guia alimentar.

Vale ressaltar que cada capítulo traz no final um resumo, para facilitar a compreensão do assunto abordado, o mesmo contém várias ilustrações coloridas e bem desenvolvidas a fim de chamar a atenção do leitor, portanto, o GAPB é acessível e com informações pontuais que abrangem vários processos do ato de alimentar-se.

\section{GUIA ALIMENTAR PARA POPULAÇÃO ARGENTINA}

A primeira edição do GAPA foi publicada no ano 2000, após quatro anos de planejamento e desenvolvimento. O processo de revisão e criação metodológica da segunda edição teve início em 2012, com a publicação em 2016. A atual edição do GAPA conta com 262 páginas e está dividido em sete capítulos: marco teórico, mensagens e gráfico, diagnóstico, objetivos nutricionais, elaboração e desenho das mensagens, validação do guia alimentar para a população, estratégia de implementação e disseminação do guia alimentar.

O primeiro capítulo, intitulado "Marco Teórico", descreve a importância e a justificativa de criação do GAPA, assim como apresenta dados referentes ao aumento das Doenças Crônicas Não Transmissíveis (DCNTs), na Argentina e no mundo, enfatizando principalmente 0 excesso de peso entre a população argentina.
Também, no primeiro capítulo, são apresentados os objetivos geral e específico, e o propósito do GAPA, que é de contribuir para mudanças nos hábitos alimentares e melhorar o estado de saúde e a qualidade de vida da população argentina. De acordo com o GAPA, a população-alvo é a população em geral maior de dois anos e que reside na Argentina, e os potenciais usuários são todas as pessoas que possam difundir os conhecimentos em educação alimentar e nutricional descritos no guia alimentar.

Além disso, no primeiro capítulo foi elaborado um resumo das etapas de construção do GAPA, assim como foi apresentado o contexto histórico de criação dos guias na Argentina e em outros países, que tiveram como marco inicial a Conferência Internacional de Nutrição, em 1992.

O segundo capítulo exibe as mensagens e o gráfico da alimentação diária. São dez mensagens que abordam a importância da variedade dos alimentos, o consumo de líquidos e o consumo moderado de sal, açúcar, gorduras e bebidas alcoólicas. As mensagens são breves e objetivas; apresentam orientações gerais para o consumo diário adequado dos indivíduos.

O gráfico da alimentação diária é apresentado ao final do segundo capítulo, sendo a roda de alimentos utilizada como representação gráfica. Os alimentos são agrupados de acordo com o grupo a que pertencem, e as divisões da roda são baseadas na ingestão diária adequada de cada grupo, com a água ao centro.

O terceiro capítulo é um diagnóstico mais detalhado dos temas que já foram abordados na introdução, como o planejamento e a organização, a população-alvo e os usuários em potencial. Exibe também as principais características dos guias da Europa e da América Latina e Caribe, que foram usados como referencial para a criação do guia argentino.

O diagnóstico, propriamente dito, é exposto a partir do tópico 3.3, em que se realiza nova busca literária e comparação de estudos sobre a situação epidemiológica, nutricional e alimentar da Argentina. Os resultados desse diagnóstico apontam para as transformações sociais e ambientais, para as mudanças nos hábitos e estilo de vida e avanços tecnológicos dos últimos anos que influenciaram diretamente o processo saúde-doença da 
população, fundamentando a justificativa e a importância de criação do GAPA para melhoria da qualidade de vida e de saúde da população.

A análise da situação epidemiológica, nutricional e alimentar identificou e quantificou os principais problemas enfrentados pela população argentina. No total foram 48 problemas estruturados em problemas relacionados ao excesso (DCNTs, elevado consumo de sal e sódio, gordura, açúcar, calorias e proteínas, entre outros) e problemas relacionados à deficiência (desnutrição, anemia, deficiência de zinco, vitamina A e B12, baixo consumo de frutas, verduras, água, pescados e carnes magras, dentre outros).

As recomendações nutricionais são apresentadas no capítulo 4, descrevendo o aporte energético diário recomendado, de $2.000 \mathrm{kcal}$, sendo $15 \%$ do Valor Energético Total (VET) de proteínas, 30\% de lipídios, $55 \%$ de carboidratos e $25 \mathrm{~g}$ de fibras alimentares. As recomendações para micronutrientes foram apresentadas em tabela. A quantidade de água recomendada ao dia foi de $2.000 \mathrm{~mL}$ para as mulheres e $2.500 \mathrm{ml}$ para os homens.

Além das recomendações, o capítulo 4 discorre sobre o plano alimentar médio, com a representação das porções adequadas de cada grupo de alimentos e sobre os principais conceitos, funções, fontes e características dos macronutrientes e micronutrientes da dieta. Ademais, explica sobre os tipos de processamento, sobre a atividade física e sobre o consumo de álcool.

Os três últimos capítulos descrevem como as recomendações, as mensagens e o gráfico foram desenvolvidos. O quinto capítulo aborda a elaboração e o desenho das mensagens do GAPA, discutindo a construção e os métodos utilizados. Assim, de acordo com o GAPA, para construção e escolha das recomendações nutricionais foi desenvolvido um método autêntico, baseado no Guia Alimentar da Guatemala e no método Angelo.

No sexto capítulo é explicada a técnica para validar o guia alimentar quanto à qualidade, à aplicabilidade e ao entendimento das mensagens e do gráfico. Para a validação interna foi desenvolvido um questionário destinado aos profissionais especialistas no tema. O questionário foi veiculado pela Federação Argentina de Graduados em Nutrição (FAGRAN). Além do questionário destinado aos especialistas, grupos focais foram selecionados para apresentar as opiniões em relação à qualidade, à transmissão e à compreensão das mensagens e do gráfico.

Após essa etapa, as mensagens e o gráfico passaram por uma segunda consulta para validação externa. Nesta etapa o objetivo era conhecer a opinião dos demais setores da população que não participaram das demais etapas.

O sétimo capítulo traz o processo de implantação do GAPA com a apresentação das estratégias utilizadas para a implementação e disseminação da nova versão do GAPA.

Ao final, percebe-se que o GAPA mesmo sendo extenso, o conteúdo foi abordado de forma objetiva e clara, com informações fundamentadas e confiáveis sobre a importância, as principais recomendações e os reflexos para a saúde da alimentação adequada e saudáveis.

\section{DISCUSSÃO}

Diversos países têm construído seus próprios guias alimentares, com objetivo semelhante, a melhoria na qualidade de vida da população em geral. Porém, cada um possui suas particularidades, considerando o perfil alimentar e nutricional da população em questão bem como questões sociais, culturais, econômicas e políticas específicas locais, podendo conter recomendações gerais distintas entre $\mathrm{si}^{ }$.

A elaboração de um guia alimentar é um processo complexo. Mais de 100 países já desenvolveram orientações oficiais sobre os alimentos, grupos de alimentos e padrões alimentares que promovem a saúde e protegem contra enfermidades ${ }^{8}$.

Tanto o Guia Alimentar para a População Brasileira quanto o Guia Alimentar para a População Argentina surgiram com a perspectiva de melhorar o padrão de consumo alimentar, pelas mudanças no perfil nutricional da população.

O Guia Alimentar para a População Brasileira é um documento com pressupostos e diretrizes para estimular as escolhas alimentares adequadas e saudáveis com vistas à prevenção de diversas doenças. A produção do GAPB é uma das estratégias para a implementação de práticas 
alimentares saudáveis e adequadas entre indivíduos e coletividades, devendo ser implementado por gestores e profissionais de saúde em parceria com atores de outros setores como educação e assistência social?.

Estudo de análise comparativa dos GAPB 2006 e 2014 enfatizou que na segunda edição os referenciais teóricos - estudos epidemiológicos, clínicos, sociológicos, antropológicos e saberes populares - e a utilização da classificação de alimentos favoreceram o desenvolvimento de diretrizes alimentares mais holísticas que abordam os padrões de alimentação e refeição, as práticas culinárias, o ato de comer e a comensalidade, possibilitando aos profissionais de saúde e a população uma compreensão do alimento enquanto parte concreta da vida dos indivíduos e coletividade, extrapolando sua dimensão fisiológica e biológica ${ }^{10}$.

A análise do guia alimentar americano destaca que este faz um apelo à ação da população deste país, incluindo três princípios norteadores: assegurar que todos os americanos tenham acesso a alimentos nutritivos e oportunidades de praticar atividade física; facilitar a mudança no comportamento individual por meio de estratégias do ambiente e definir o cenário para uma alimentação saudável ao longo da vida, atividade física e manutenção do peso ${ }^{7}$.

De acordo com os dados relatados no guia argentino (2016), em 2005, 49,1\% da população argentina apresentavam excesso de peso, em 2009, eram $53,4 \%$ e em 2013, a mesma tendência de crescimento foi observada. No Brasil dados da Pesquisa Nacional de Saúde (2014) apontam que, em 2013, 56,9\% dos adultos com idade superior a 18 anos apresentam excesso de peso e $20,8 \%$ já se encontram na faixa de obesidade, afetando especialmente as mulheres, que apresentam prevalências de excesso de peso e de obesidade maiores que as dos homens ${ }^{11}$.

Com essas mudanças, cada país buscou de forma particular identificar os principais pontos e construir um guia alimentar que promova saúde e bem-estar de modo geral a partir de práticas diárias. Com a leitura dos guias foi possível destacar alguns pontos, como apresentado nos Quadros 1, 2 e 3 que sintetizam a comparação destes, quanto à estrutura e ao desenvolvimento.
Quadro 1. Principais diferenças entre o Guia Alimentar Brasileiro e Argentino, 2019

\begin{tabular}{|c|c|c|}
\hline 丞 & BRASILEIRO & ARGENTINO \\
\hline $\begin{array}{l}\text { 胥 } \\
\text { 恶 } \\
\text { 恶 }\end{array}$ & 158 páginas & 262 páginas \\
\hline 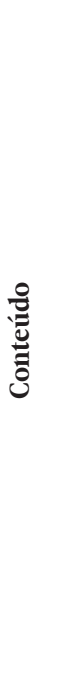 & $\begin{array}{l}\text { Preâmbulo } \\
\text { Introdução } \\
\text { 1. Princípios } \\
2 \text {. A escolha dos alimentos } \\
\text { 3. Dos alimentos à refeição } \\
4 \text {. O ato de comer e a } \\
\text { comensalidade } \\
\text { 5.A compreensão e a superação } \\
\text { de obstáculos uma } \\
\text { Dez passos para uma adequada e } \\
\text { alimentação } \\
\text { saudável } \\
\text { Para saber mais } \\
\text { Anexos }\end{array}$ & $\begin{array}{l}\text { 1. Marco teórico } \\
\text { 2. Mensagens e gráfico } \\
\text { 3. Diagnóstico } \\
\text { 4. Objetivos nutricionais } \\
\text { 5. Elaboração e desenho } \\
\text { das mensagens } \\
\text { 6. Validação do guia para } \\
\text { população } \\
\text { 7. Estratégia de } \\
\text { implementação do guia } \\
\text { disseminação do } \\
\text { alimentar }\end{array}$ \\
\hline 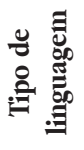 & Acessível, simples e direta & Mista (técnica e Aaessível) \\
\hline
\end{tabular}

Fonte: Dados do estudo, Teresina - 2019.

Quadro 2. Pontos positivos e negativos do Guia Alimentar Brasileiro e Argentino, 2019

\begin{tabular}{|c|c|c|}
\hline 莺 & BRASILEIRO & ARGENTINO \\
\hline 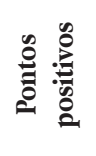 & $\begin{array}{l}\text { Fácil entendimento } \\
\text { Ilustrativo } \\
\text { Divisão adequada }\end{array}$ & $\begin{array}{l}\text { Informações completas } \\
\text { Conteúdo bem elaborado }\end{array}$ \\
\hline : & $\begin{array}{l}\text { Baixa distribuição para a } \\
\text { população e poucos dados } \\
\text { científicos. }\end{array}$ & $\begin{array}{l}\text { Conteúdo científico em } \\
\text { excesso e gráficos de difícil } \\
\text { entendimento }\end{array}$ \\
\hline
\end{tabular}
Fonte: Dados do estudo, Teresina - 2019.abordagem da promoção de práticas alimentares saudáveis 
Quadro 3. Promoção de práticas alimentares saudáveis do Guia Alimentar Brasileiro e Argentino, 2019

\begin{tabular}{|c|c|c|}
\hline 莺 & BRASILEIRO & ARGENTINO \\
\hline 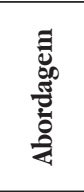 & $\begin{array}{l}\text { Promoção de alimentação } \\
\text { adequada e saudável e } \\
\text { incentivo à autonomia nas } \\
\text { escolhas dos alimentos. }\end{array}$ & $\begin{array}{l}\text { Diagnósticos e caracterização } \\
\text { do perfil atual da dieta da } \\
\text { população e recomendações } \\
\text { sobre alimentação e práticas } \\
\text { saudáveis. }\end{array}$ \\
\hline 营 & $\begin{array}{l}\text { Recomendações gerais sobre } \\
\text { a escolha de alimentos com } \\
\text { textos e imagens, exemplos de } \\
\text { refeições e ato de comer. }\end{array}$ & $\begin{array}{l}\text { Mensagem e gráfico } \\
\text { da alimentação diária, } \\
\text { recomendações alimentar e } \\
\text { nutricional com dados. }\end{array}$ \\
\hline
\end{tabular}

Fonte: Dados do estudo, Teresina - 2019.

Ao comparar o tamanho dos guias alimentares, percebe-se que o guia argentino é mais extenso, com 262 páginas, apresentando mais conteúdo textual e visual, porém o tamanho da letra é reduzido quando comparada à letra usada no guia brasileiro. O GAPB contém 158 páginas, com informações mais pontuais e objetivas, que facilita a leitura e a compreensão pela população em geral.

Apesar da linguagem mais técnica o guia argentino não utiliza expressões que condenem alimentos, evitando impor recomendações, e enfatizando palavras como "prefira" "diminua", "limite", "escolha", "se possível", assim como no brasileiro, referenciando de forma positiva vantagens e benefícios da alimentação saudável.

De acordo com a Food and NutritionBoard/ World Health Organization (FNB/WHO) ${ }^{12}$, os guias dietéticos baseados nos alimentos têm como objetivo favorecer a educação nutricional a partir de termos que sejam compreensíveis, simples e claros para a maioria dos consumidores, e que indiquem as modificações necessárias nos padrões alimentares de uma dada população rural e urbana e mesmo de grupos específicos.

O conteúdo dos guias é direcionado para a população em geral, porém o argentino apresenta, no decorrer do texto, conteúdo científico, tabelas, gráficos, e fluxogramas, que dificultam o entendimento da população leiga. Visto isso, foi elaborado recentemente um "manual de aplicação" com linguagem mais simplificada e direcionada tanto a equipes de saúde quanto à comunidade em geral. O GAPA não faz menção à alimentação e valorização dos alimentos regionais, o mesmo traz o gráfico da alimentação diária, como a forma de representação da recomendação dos grupos de alimentos.

Quanto ao guia brasileiro, o conteúdo apresentado é sucinto, com linguagem acessível e ilustrações autoexplicativas, o que pode estar atribuído à atualização de 2014, que teve como objetivo facilitar a compreensão por todas as pessoas sobre alimentação saudável e Segurança Alimentar e Nutricional (SAN), além de considerar a cultura local. O GAPB ainda possui referência com fotos de diferentes preparações típicas das regiões brasileiras, apesar de não possuir as porções de alimentos.

Uma análise de Almeida et al. $^{8}$ conseguiu identificar o que é uma alimentação saudável e de risco para o desenvolvimento de doenças, por meio das representações gráficas; o mesmo enfatiza que as representações gráficas exercem papel primordial na complementação dos guias alimentares, auxiliando o público-alvo a recordar facilmente os alimentos que devem ser consumidos.

O guia argentino possui dez mensagens, semelhantes aos dez passos para uma alimentação adequada e saudável do guia brasileiro. Cada mensagem possui submensagens com indicações de como e o porquê das escolhas alimentares, incentivando uma alimentação em local tranquilo e em companhias agradáveis, assim como traz o guia brasileiro, todavia o GAPA apresenta essas informações de forma mais sucinta.

Em relação à abordagem direcionada para adolescentes, público-alvo do projeto vinculado à Rede SSAN, parceria entre o Brasil e Argentina, percebe-se que o guia brasileiro apresenta mais recomendações direcionadas a esse grupo, como realizar uma ou mais pequenas refeições, comer em companhia de adultos, envolver-se na compra de alimentos e no preparo das refeições. No guia argentino são poucas as recomendações voltadas aos adolescentes, em contrapartida o GAPA traz mais informações sobre esses indivíduos, caracterizando a dieta atual e apresentando seus principais fatores determinantes.

O número de crianças e adolescentes (de 05 a 19 anos) obesos em todo o mundo aumentou dez vezes 
nas últimas quatro décadas. De acordo com um novo estudo liderado pelo Imperial College London e pela Organização Mundial da Saúde (OMS) se as tendências atuais continuarem haverá mais crianças e adolescentes com obesidade do que com desnutrição moderada e grave até $2022^{13}$. Algumas mudanças nos hábitos de vida dos adolescentes refletem esses dados como, por exemplo, hábitos alimentares pouco saudáveis, comer fora de casa e a substituição de refeições tradicionais por lanches, o que leva ao consumo de alimentos de alta densidade energética, rico em gorduras e carboidratos simples ${ }^{14}$.

O Guia Alimentar para a População Brasileira relaciona a promoção da saúde com as dimensões de produção, acesso/comercialização, processamento e consumo de alimentos na proposta de aproximação entre produtor e consumidor, por estímulo da aquisição em feiras e outros mercados institucionais, nas práticas alimentares de base agroecológica, e no resgate dos saberes tradicionais de produção e processamento de alimentos pela agricultura familiar ${ }^{15}$.

O guia alimentar estabelece um conjunto de diretrizes alimentares para os brasileiros, com base no cenário epidemiológico e evidências científicas; seu principal objetivo é orientar práticas alimentares que visem à promoção da saúde e à prevenção de doenças relacionadas à alimentação $0^{16}$.

A educação alimentar e nutricional quando aliada à produção alimentar sustentável promove conhecimentos e possibilita escolhas que melhoram a utilização e o consumo dos alimentos, assim como a melhoria na qualidade de vida da população. ${ }^{17} \mathrm{~A}$ educação alimentar e nutricional deve ser composta por ações coordenadas, sendo mais que um instrumento, e envolvendo diferentes setores e ensinamentos com a transmissão de informações por meio de técnicas como palestras, produção de materiais informativos, dentre outros $^{18}$.

A abordagem prática que visam a prevenção e a promoção de saúde, por meio da educação alimentar e nutricional, foi observada nos dois guias. O brasileiro optou por assuntos mais práticos do dia a dia, com exemplos e imagens de como fazer, sendo uma ferramenta útil tanto para a população em geral, como para atividades educativas.
Estudos desenvolvidos que utilizam os dez passos para uma alimentação adequada e saudável, ferramenta apresentada no GAPB, em ações de educação alimentar e nutricional têm demonstrado efeito positivo na promoção da saúde ${ }^{19,20}$.

Com relação ao guia argentino, de acordo com Demonte ${ }^{21}$, o guia propõe-se a traduzir as recomendações nutricionais de caráter técnico em mensagens objetivas, de linguagem simples e fazendo uso do gráfico para facilitar compreensão, a fim de contribuir com a mudança nos hábitos alimentares da população argentina que proporcionam a melhora da saúde e da qualidade de vida.

O guia alimentar brasileiro tem sido considerado uma importante referência entre os guias alimentares atuais, por valorizar questões como comer em companhia, diversidade cultural e, especialmente, o posicionamento político que defende ao recomendar o uso limitado de alimentos ultraprocessados, levando em conta as evidências científicas atuais?

Possivelmente baseado no GAPB, o Canadá lançou no início de 2019 seu guia dietético para profissionais de saúde e legisladores políticos com o objetivo de promover uma alimentação saudável e bem-estar geral, apoiando melhorias para o ambiente alimentar canadense ${ }^{22}$.

Visto que uma das inovações do GAPB foi trabalhar com a classificação dos alimentos segundo seu nível de processamento ${ }^{15}$, o Canada's food guide aborda os alimentos que devem ser minimizados ou evitados (bebidas e carnes processadas), pelo potencial impacto negativo à saúde com o consumo regular desses alimentos. Já a inovação do guia canadense foi recomendar a troca, quando possível, de carnes vermelhas por proteínas vegetais, incentivando o consumo de gorduras saudáveis ao invés de gorduras saturadas.

Correlacionando os dois guias, o brasileiro apresentou mais estratégias didáticas que facilitam o entendimento como resumos ao final de cada capítulo, ilustrações e fotos, letra legível, entre outros. O guia argentino traz recomendações simples de entender e diretas, além de apresentar uma linguagem visual, com o gráfico, sintetizando as recomendações, porém, os demais conteúdos são extensos e deixam a leitura pouco objetiva. 


\section{CONCLUSÃO}

A construção de um guia alimentar abrange várias etapas, e para o desenvolvimento é levado em consideração as questões sociais, culturais, econômicas, políticas, entre outras, de cada país. Ao comparar o Guia Alimentar para a População Brasileira e o Guia Alimentar para a População Argentina foi possível perceber que as diferenças entre os guias estão, apenas, na estrutura e na abordagem. Sendo a promoção à saúde, por meiode hábitos alimentares saudáveis, o foco principal dos guias, com cada um alcançando seus objetivos individualmente.

Estudos como este promovem discussões críticas sobre a construção e aplicação de guias alimentares, além de produzirem mais conhecimento sobre educação alimentar e nutricional e incentivarem a busca e 0 desenvolvimento de estudos sobre esta temática. A melhoria da qualidade e o aprimoramento dos guias alimentares são fundamentais para a prevenção de doenças e a promoção de saúde. Com isso, estudos que abordem o entendimento e o ponto de vista da população sobre o guia alimentar fazem-se necessários.

\section{REFERÊNCIAS}

1. Barbosa RMS, Colares LGT, Soares EA. Desenvolvimento de guias alimentares em diversos países Development of food-based dietary guidelines in several countries. Rev Nutr. 2008;21(4):455-467.

2. Global Strategyon Diet, PhysicalActivity and Health. [internet] 2015 [acesso em 2018 dez. 05]. Disponível em: http://www.who.int/ dietphysicalactivity/strategy/eb11344/strategy_ english_web.pdf.

3. Brasil. Ministério da Saúde. Guia Alimentar para População Brasileira: promovendo alimentação saudável. Brasília: Ministério da Saúde. [internet] 2008 [acesso em 2018 dez. 19]. Disponível em: http://www.sonutricao.com.br/downloads/Guia Alimentar_Populacao_Brasileira.pdf.

4. Brasil, Departamento de Atenção Básica, Secretaria de Atenção à Saúde, Ministério da Saúde. Guia alimentar para a população brasileira. 2 Ed. Brasília:
Ministério da Saúde; 2014.

5. Argentina, Ministerio de Salud de la Nación. Guías Alimentarias para laPoblación Argentina. Buenos Aires, 2016.

6. Wendpap LL, Ferreira MG, Rodrigues PRM, Pereira RA, Loureiro AS, Silva RMVG. Qualidade da dieta de adolescentes e fatores associados. Cad Saúde Pública. 2014;30:97-106.

7. Andrade LM, Bocca C. Análise comparativa de guias alimentares: proximidades e distinções entre três países. DEMETRA. 2016;11(4):1001-1016.

8. Almeida CJ, Rochedo CAL, Maria ISS, Mariane TO. Guia alimentar para a população brasileira: complementação por meio de representações gráficas. RBONE. 2018;12(71):316-328.

9. Goiás. Governo do Estado. Secretaria de Estado da Saúde; Superintendência de Vigilância em Saúde; Coordenação de Vigilância Nutricional. Material de apoio: Guia Alimentar para a População Brasileira na prática das equipes de saúde, educação, e assistência social em Goiás. Goiânia: SUVISA/GVE/ CVN, 2016.

10. Oliveira MSS, Santos LA. Guias alimentares para a população brasileira: Uma análise a partir das dimensões culturais e sociais da alimentação. Ciênc Saúde Colet [internet] 2018. [acesso em 2019 jul. 25]. Disponível em: http://www. cienciaesaudecoletiva.com.br/artigos/guiasalimentares-para-a-populacao-brasileira-umaanalise-a-partir-das-dimensoes-culturais-e-sociais-daalimentacao/16986.

11. Instituto Brasileiro de Geografia e Estatística. Pesquisa Nacional de Saúde 2013: percepção do estado de saúde, estilos de vida e doenças crônicas. Rio de Janeiro: IBGE. 2014 [acesso em 2019 jan. 12]. Disponível em: ftp://ftp.ibge.gov.br/PNS/2013/ pns2013.pdf.

12. Food and NutritionBoard/World Health Organization. Preparation and use of food based dietary guidelines. Geneva, 1996. Report of joint FAO/WHO consultation.

13. Organização Pan-Americana da Saúde; Organização Mundial da Saúde. Obesidade entre crianças e adolescentes aumentou dez vezes em quatro 
décadas, revela novo estudo do Imperial College

Recebido em:23/03/2019

London e da OMS. [internet] 2017 [acesso em 26

Aceito em: 20/08/2019

jan. 19]. Disponível em: https://www.paho.org/bra/ index.php?option $=$ com_content\&view $=$ article $\&$ id $=5527$ :obesidade-entre-criancas-e-adolescentesaumentou-dez-vezes-em-quatro-decadas-revelanovo-estudo-do-imperial-college-london-e-daoms\&Itemid $=820$.

14. Poti JM, Duffey KJ, Popkin BM. The association of fast food consumption with poor dietary outcomes and obesity among children: is it the fast food or the remainder of the diet. Am J Clin Nutr. 2014;99(1):162-71.

15. Oliveira NRF, Jaime PC. O encontro entre 0 desenvolvimento rural sustentável e a promoção da saúde no Guia Alimentar para a População Brasileira. Saúde Soc. 2016; 25(4):1108-1121.

16. Barbosa FS, Sichieri R, Junger WL. Assessing usual dietary intake in complex sample design surveys: the National Dietary Survey. Rev Saúde Pública. 2013;47:171-176.

17. Marin T, Berton P, Santo LKRS. Educação nutricional e alimentar: por uma correta formação dos hábitos alimentares. Rev F@pciência. 2009;3(7):72-78.

18. Santos LAS.O fazer educação alimentar e nutricional: algumas contribuições para reflexão. Ciênc Saúde Colet. 2012;17(2):453-462.

19. Schirmer CL, Seibel R, Deon RG, Schwanke CHA, Silva Filho IG, Bós AJG. Adesão aos dez passos para uma alimentação saudável em pessoas idosas. Saúde e Pesqui. 2017;10(3):501-509.

20. Almeida AA, Maia DERS, Oliveira EIAD, Cardoso IB, Araújo JO, Navarro LN, et al. Ação de educação alimentar e nutricional para funcionários de um hospital universitário: um relato de experiência. Gepnews. 2018;1(4):2-12.

21. Demonte FC. Recomendaciones y consejosalimentarios. Análisis de losmensajesaudiovisuales que difunde el estado argentino. Question. 2017;1(53):236-255.

22. Canada. Health Canada. Canada's Dietary Guidelines for healthprofessionals and policymakers. Ottawa, 2019. 